\title{
Burst-Mode Clock and Data Recovery in Optical Multiaccess Networks Using Broad-Band PLLs
}

\author{
Alan Li, Student Member, IEEE, Julien Faucher, Student Member, IEEE, and David V. Plant, Senior Member, IEEE
}

\begin{abstract}
Broad-band phase-locked loops (PLLs) are proposed for burst-mode clock and data recovery in optical multiaccess networks. Design parameters for a charge-pump PLL-based clock and data recovery (CDR) with fast phase acquisition are derived using a time-domain model that does not assume narrow loop bandwidth or small phase errors. Implementation in a half-rate CDR circuit confirms a clock phase acquisition time of $40 \mathrm{ns,} \mathrm{or}$ 100 bits at $2.488-\mathrm{Gb} / \mathrm{s}$ rate, and data recovery at $1.244-\mathrm{Gb} / \mathrm{s}$ rate with a bit-error rate of $1 \times 10^{-10}\left(2^{14}-1\right.$ pseudorandom binary sequence with Manchester-encoding). The CDR was fabricated in complementary metal-oxide-semiconductor $0.18-\mu \mathrm{m}$ technology in an area of $1 \times 1 \mathrm{~mm}^{2}$ and consumes $54 \mathrm{~mW}$ of power from a 1.8-V supply.
\end{abstract}

Index Terms-Burst-mode receivers, clock and data recovery (CDR), half-rate clock and data recovery (CDR), multiaccess communication, optical communication, phase-locked loops (PLLs).

\section{INTRODUCTION}

D EMANDS for broad-band access are predicted to exceed the potential of modern networks. Optical multiaccess networks based on an all-optical core, such as passive optical network, have been proposed to enable services such as IP telephony and video on demand in metro and access areas [1], [2]. Technologies focusing on this all-optical core are already mature [3]. However, novel electronics are also required to support new functionalities at the network edges. Specifically, optical receivers must be adapted to deal with burst-mode traffic, where data bursts originate from various sources and travel different distances due to the point-to-multipoint nature of the network. The amplitude and phase of successive packets may therefore vary anywhere between $0-20 \mathrm{~dB}$ and $-\pi$ to $+\pi \mathrm{rad}$, respectively [4]. Receivers with the following characteristics are therefore necessary: wide dynamic range, fast automatic threshold control (ATC), and fast phase acquisition, where ATC and phase acquisition must occur within nanoseconds to support short packet lengths at gigabit rates. Burst-mode receiver front-ends have already been demonstrated with wide dynamic range and fast ATC [5]. This work focuses on the fast phase acquisition requirement. Whereas previous works have considered correlation algorithms [6] and gated oscillators [7] to decrease phase acquisition time in clock and data recovery (CDR), this work investigates the use of broad-band phase-locked loops (PLLs). While it is known that increasing the bandwidth of a PLL can tradeoff jitter performance in favor of phase/frequency acquisition time

Manuscript received August 29, 2005; revised September 28, 2005. This work was supported by industrial and government partners through the Agile AllPhotonic Networks (AAPN) Research Network, and by the CMC.

The authors are with the Photonics Systems Group, Electrical and Computer Engineering Department, McGill University, Montreal, QC H3A 2A7, Canada (e-mail: alanli@photonics.ece.mcgill.ca; julienf@ photonics.ece.mcgill.ca).

Digital Object Identifier 10.1109/LPT.2005.860396
[8], this work addresses practical issues in applying this concept to burst-mode CDR. We demonstrate successful implementation of a burst-mode broad-band PLL CDR through modeling and experimentation, and quantify the resulting tradeoff on line coding. In particular, the phase acquisition time of a broad-band PLL CDR is measured and the effect of various coding schemes on the phase noise of the recovered clock is tested. Section II discusses the advantages and tradeoffs of broad-band PLL CDRs. Section III describes modeling and implementation, while Section IV describes the experimental results.

\section{BROAD-BAND PLL CDR}

SONET CDRs are specified with a narrow PLL bandwidth in order to minimize jitter accumulation through long repeater chains in long-haul networks [9]. This is because narrow-band PLLs are highly resistant to phase variations which cause jitter. However, this optimizes SONET CDRs for suppression of data jitter at the expense of other abilities: suppression of internal jitter from the voltage-controlled oscillator ( $\mathrm{VCO}$ ), frequency capture range, and phase/frequency acquisition time. This last tradeoff is central to the discussion. A narrow-band PLL's resistance to phase variations implies a slow acquisition time to steps in the incoming phase. The phase acquisition time of a commercially available SONET OC-48 CDR was previously measured at 1400 bits ( $\pm \pi$-rad phase steps) [10], which translates to microseconds at gigabit rates. In contrast to SONET networks, optical multiaccess networks are typically deployed in metropolitan areas where few or no repeaters are necessary. This opens the possibility of relaxing the restriction on the PLL's loop bandwidth.

We therefore consider a broad-band PLL CDR to achieve fast phase acquisition in optical multiaccess networks. Specifically, we modify the PLL parameters in a SONET CDR to increase loop bandwidth until a target phase acquisition time is obtained. Theoretically, this has the added benefits of increasing the CDR's frequency capture range and suppression of internal jitter, as the PLL behaves as a high-pass filter for jitter generated by the VCO [8]. However, as PLLs are unable to distinguish a true phase-step from cycle-to-cycle data jitter, increasing the loop bandwidth simultaneously increases the jitter transfer bandwidth. Also, broad-band PLLs are more prone to clock drift when receiving data with low transition-density, making line coding necessary to eliminate low frequency components. Therefore, the tradeoffs are reduced suppression of data jitter and a strong requirement for line coding. We quantify these tradeoffs below.

\section{MODELING AND IMPLEMENTATION}

Although they are of higher order and complexity, chargepump PLL-based CDRs are often simplified to second-order 


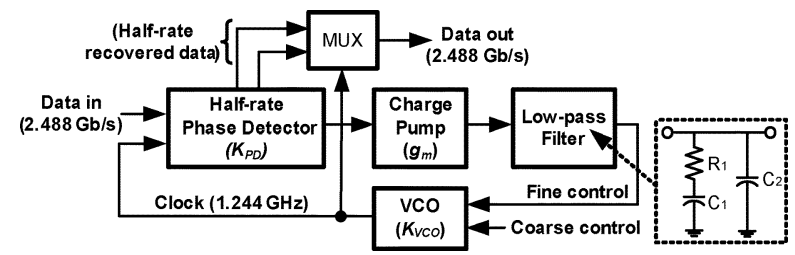

Fig. 1. Half-rate CDR architecture [11], targeting a 2.488-Gb/s bit rate.

models used in the frequency-domain. However, their accuracy relies heavily upon two assumptions [11]: 1) the instantaneous phase error $\left(\varphi_{e}\right)$ between the input and the recovered clock is small, which restricts the model to cases where the system does not deviate far from steady-state; and 2) the loop bandwidth $(K)$ is a small fraction of the input frequency $(\gg 10 \cdot K[11])$, which allows a continuous-time approximation of the loop's behavior where digital switching granularity is ignored in favor of the average behavior over many cycles. The two assumptions produce a linear baseband model of charge-pump PLLs to facilitate analysis. However, these assumptions do not apply in the present case. Another consideration is that stability becomes an issue as a PLL's bandwidth is increased. This is especially true for charge-pump PLLs, which are time-variant due to the discontinuous sampling operation [11]. A more reliable model is therefore necessary for this application, particularly when modeling responses to large phase steps.

An alternative mathematical model for charge-pump PLLs was proposed in [11] and [12]. The model is based on a system of nonlinear difference equations that account for the highly nonlinear nature of PLLs directly in the time-domain. It therefore places no restrictions on $K$ or $\varphi_{e}$ to produce transient responses to phase steps. The model was used here to derive component values which achieve fast phase acquisition in a half-rate CDR architecture intended for SONET [8] (Fig. 1). Specifically, parameters for a second-order loop filter $\left(R_{1}, C_{1}, C_{2},\right)$ and linear gains for the phase detector $\left(K_{\mathrm{PD}}[\mathrm{V} / \mathrm{rad}]\right)$, charge-pump $\left(g_{m}[\mathrm{~A} / \mathrm{V}]\right)$, and $\mathrm{VCO}$ $\left(K_{\mathrm{VCO}}[\mathrm{Hz} / \mathrm{V}]\right)$ were derived for a target clock frequency of $1.244 \mathrm{GHz}$ (Table I). Fig. 2 shows the resulting transient responses (VCO control voltage) to phase steps within the range $[0, \pm \pi / 2] \mathrm{rad}$, which were generated using the model. Note that the input is a periodic signal resembling a burst preamble and that frequency-lock is assumed as an initial condition. Stability is verified over all simulated phase steps. In all cases, the CDR requires $40 \mathrm{~ns}$ to settle to within approximately $10 \%$ of the steady-state value, corresponding to 100 bits at $2.488-\mathrm{Gb} / \mathrm{s}$ data rate. In comparison, [8] exhibited a 350-ns lock time. To quantify the jitter tradeoff, the analysis and equations from [8, Ch. 5] were used to derive the loop bandwidth and jitter peaking resulting from the design parameters of Table I. $K$ was calculated to be $5.78 \times 10^{8} \mathrm{rad} / \mathrm{s}$, or $92 \mathrm{MHz}$, while jitter peaking was $1.202 \mathrm{~dB}$. These results denote a wider jitter transfer bandwidth and higher peaking than specified in ITU/Bellcore specifications for SONET OC-48 $(2 \mathrm{MHz}$ and $0.1 \mathrm{~dB}$, respectively [9]). This confirms that a broad-band PLL CDR has been achieved resulting in a tradeoff in data jitter-suppression abilities. Also, the ratio of clock frequency over $K$ is only $\sim 13.5$, indicating that performance assessments from a baseband frequency-domain model would not be reliable [11].

The derived PLL parameters were implemented in the CDR architecture from [8], targeting a data rate of $2.488 \mathrm{~Gb} / \mathrm{s}$. The
TABLE I

Circuit Design Parameters Obtained From Simulation

\begin{tabular}{cc}
\hline \hline \multirow{2}{*}{ Parameter } & Value \\
\hline$R_{l}$ & $5 \mathrm{~K} \Omega$ \\
$C_{l}, C_{2}$ & $2.5 \mathrm{pF}, 300 \mathrm{fF}$ \\
$K_{P D}$ & $25 \mathrm{mV} / \mathrm{rad}$ \\
$g_{m}$ & $400 \mu \mathrm{A} / \mathrm{V}$ \\
$K_{V C O}$ & $2.0 \mathrm{GHz} / \mathrm{V}$ \\
$\left.I_{P}=K_{P D} \cdot g_{m}\right)$ & $10 \mu \mathrm{A} / \mathrm{rad}$ \\
\hline
\end{tabular}

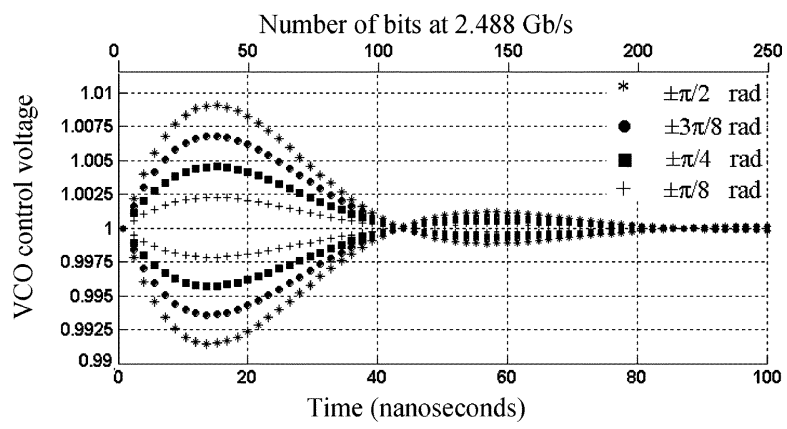

Fig. 2. Transient simulation from nonlinear time-domain model of VCO control voltage with respect to time and number of bits of incoming data (2.488 $\mathrm{Gb} / \mathrm{s})$ in response to phase steps of $\pm \pi / 8, \pm \pi / 4, \pm 3 \pi / 8$, and $\pm \pi / 2 \mathrm{rad}$.

architecture is a half-rate CDR which samples data on both the rising and falling edges of the clock. Such architectures use a VCO running at half the data rate to facilitate implementation in complementary metal-oxide-semiconductor (CMOS), and offer significant advantages in the proposed application. Following a phase step, the worst-case clock realignment is $\pm \pi / 2 \mathrm{rad}$, as opposed to $\pm \pi$ rad for a full-rate architecture. Also, each recovered bit occurring on the negative-edge of the clock would not be sampled by subsequent positive-edge triggered systems. Therefore, in the case of Manchester-encoded data, this would automatically recover the original uncoded data if made to positively trigger on the correct cycle of bits.

The phase detector was implemented using CML logic to reduce switching noise and increase speed. The gains of the implemented phase detector, charge-pump, and VCO were $23.56 \mathrm{mV} / \mathrm{rad}, 395 \mu \mathrm{A} / \mathrm{V}$, and $1.93 \mathrm{GHz} / \mathrm{V}$, respectively. In order to observe phase acquisition, the differential control voltage of the VCO was brought off-chip using a buffer, to minimize loading effects, and an amplifier. This added significant power consumption but enabled observation of the internal PLL behavior during transients. Fifty-Ohm low-voltage differential signal input-output buffers were used to bring high-speed signals on- and off-chip.

\section{EXPERIMENTAL RESULTS}

The circuit was fabricated in CMOS $0.18-\mu \mathrm{m}$ in an area of $1 \times 1 \mathrm{~mm}^{2}$. The die was packaged in a 24-pin open-cavity ceramic package mounted on a custom designed FR4 printed circuit board. The measured power consumption was $54 \mathrm{~mW}$ from a 1.8-V power supply. The VCO could operate anywhere between $622 \mathrm{MHz}$ and $1.244 \mathrm{GHz}$ using coarse tuning.

The phase acquisition time of the CDR was measured using a repeating $2.488-\mathrm{Gb} / \mathrm{s}$ pattern consisting of 32 consecutive zeros, representing a period of nontransmission, followed by a 112-bit "1010..." pattern, representing the preamble of a burst packet. 


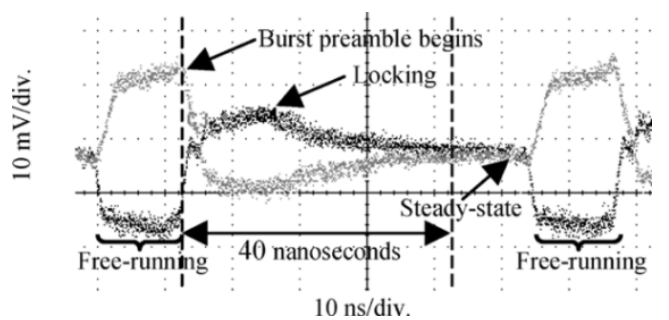

Fig. 3. Scope trace of differential VCO control signal from implemented circuit in response to data burst preamble.

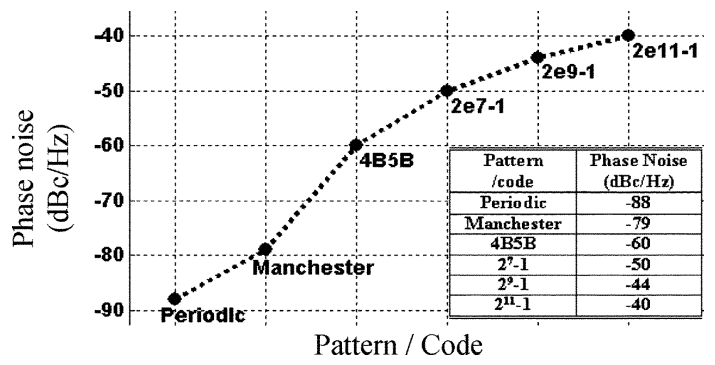

Fig. 4. Phase noise measured at $1-\mathrm{MHz}$ offset from peak for various data patterns of decreasing transition density.

Fig. 3 depicts a scope trace of the PLL's response (VCO control voltage). Note that the trace is an amplified version of the control voltage. The periods of nontransmission and preamble are each evident in the trend. During periods of nontransmission, the system is free-running and the phase of the VCO is random with respect to incoming data. The CDR begins locking at the beginning of the preamble and reaches steady-state within approximately $40 \mathrm{~ns}$, or 100 bits at $2.488 \mathrm{~Gb} / \mathrm{s}$. The trend and timescale of the CDR response are comparable to simulation results (Fig. 2), which verifies the accuracy of the PLL model proposed in [10]. It also confirms that a greater than ten-fold improvement in phase acquisition time has been achieved over that of a SONET CDR [10].

The CDR was then tested with various line coding schemes. As expected, a strong dependence on transition density was observed (Fig. 4). Only Manchester-encoding resulted in a recovered clock of sufficient quality for bit-error-rate (BER) testing. Manchester-encoded pseudorandom binary sequence patterns of length $2^{7}-1,2^{11}-1$, and $2^{14}-1$ were used to make BER measurements. Error-free data recovery could not be achieved at $2.488 \mathrm{~Gb} / \mathrm{s}$ due to poor performance of the data output buffers, which resulted in attenuation. The CDR was therefore tested at $1.244 \mathrm{~Gb} / \mathrm{s}$ with a recovered half-rate clock of $622 \mathrm{MHz}$. This produced a baud rate of $1.244 \mathrm{~Gb} / \mathrm{s}$ and an effective recovered bit rate of $622 \mathrm{Mb} / \mathrm{s}$ due to Manchester-encoding. BER measurements were performed by loading Manchester-encoded patterns into the pattern generator and the corresponding uncoded patterns into the error detector. This resulted in a BER of $1 \times 10^{-10}$ for all three patterns. Fig. 5 depicts a persistence scope trace of the recovered data. The small vertical eye opening $(50 \mathrm{mV})$ is caused by attenuation by the data output buffers. Fig. 6 depicts the recovered clock spectrum for a Manchester-encoded pattern. A phase noise of $-79 \mathrm{dBc} / \mathrm{Hz}$ was measured at a $1-\mathrm{MHz}$ offset.

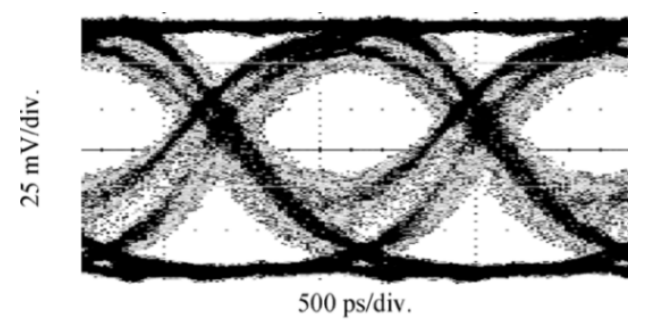

Fig. 5. Recovered data eye diagram (20 s persistence).

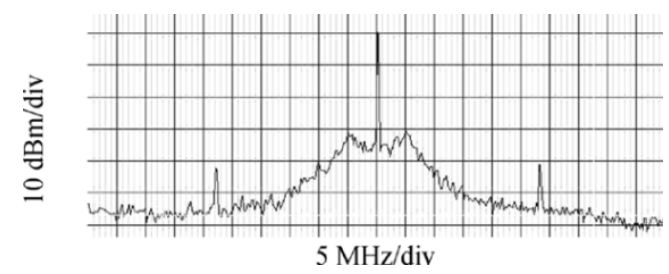

Fig. 6. Spectrum of recovered clock, 100-MHz span (peak $-17 \mathrm{dBm}$ at $622 \mathrm{MHz})$.

\section{CONCLUSION}

We have designed a broad-band PLL CDR for burst-mode applications. A time-domain model was used to design the CDR. The model was verified experimentally and a phase acquisition time of 100 bits was measured. This represents a greater than ten-fold improvement over corresponding SONET CDRs. Successful CDR was demonstrated and the tradeoff between lock acquisition time and PLL bandwidth resulted in the need for Manchester encoding. This work may be scaled to match the lock time and coding requirements of specific networks.

\section{REFERENCES}

[1] M. Nakamura et al., "Proposal of networking by PON technologies for full and ethernet services in FTTx," J. Lightw. Technol., vol. 22, no. 11, pp. 2631-2640, Nov. 2004.

[2] Y. Maeda, K. Okada, and D. Faulkner, "FSAN OAN-WG and future issues for broadband optical access networks," IEEE Commun. Mag., vol. 39, no. 12, pp. 126-132, Dec. 2001.

[3] Y.-L. Hsueh et al., "A highly flexible and efficient passive optical network employing dynamic wavelength allocation," J. Lightw. Technol., vol. 23, no. 1, pp. 277-286, Jan. 2005.

[4] S. Chao, L.-K. Chen, and K.-W. Cheung, "Theory of burst-mode receiver and its applications in optical multiaccess networks," J. Lightw. Technol., vol. 15, no. 4, pp. 590-606, Apr. 1997.

[5] S. Vatannia, P.-H. Yeung, and C. Lu, "A fast response $155-\mathrm{Mb} / \mathrm{s}$ burstmode optical receiver for PON," IEEE Photon. Technol. Lett., vol. 17, no. 5, pp. 1067-1069, May 2005.

[6] C. A. Eldering et al., "Digital burst mode clock recovery technique for fiber-optic systems," J. Lightw. Technol., vol. 12, no. 2, pp. 271-279, Feb. 1994.

[7] Y. Ota et al., "High-speed, burst-mode, packet-capable optical receiver and instantaneous clock recovery for optical bus operation," J. Lightw. Technol., vol. 12, no. 2, pp. 325-331, Feb. 1994.

[8] J. Savoj and B. Razavi, High-Speed CMOS Circuits for Optical Receivers. Norwell, MA: Kluwer, 2001, pp. 25-26, 76-93.

[9] ITU/Bellcore, GR-253-CORE.

[10] J. Faucher, M. Mony, and D. V. Plant, "Test setup for optical burst-mode receivers," in Proc. IEEE Lightwave Technologies in Instrumentation and Measurement Conf., 2004, pp. 123-128.

[11] F. M. Gardner, "Charge-pump phase-lock loops," IEEE Trans. Commun., vol. 28, no. 11, pp. 1849-1858, Nov. 1980.

[12] M. Van Paemel, "Analysis of a charge-pump PLL: a new model," IEEE Trans. Commun., vol. 42, no. 7, pp. 2490-2498, Jul. 1994. 